an encouraging desire for education and skill enhancement. Refinement of the tool, further exploration of the views of RPs is planned in order to tailor educational strategies.

\title{
80 ADVANCE CARE PLANNING BY RESPIRATORY PHYSICIANS WITH PATIENTS WITH COPD. A PILOT SURVEY
}

TA Smith',2,3, M Kim¹,2, M Piza1,2, PM Davidson4, CR Jenkins5, JM Ingham¹,2, JM Clayton ${ }^{6}$ Cunningham Centre For Palliative Care, Sydney, Australia; ${ }^{2}$ The University of New South Wales, Faculty of Medicine, Sydney, Australia; ${ }^{3}$ The Campbell town Hospital, Sydney, Australia; ${ }^{4}$ University of Technology, Faculty of Nursing, Sydney, Australia; ${ }^{5}$ The Concord General and Repatriation Hospital, Sydney, Australia; ${ }^{6}$ Hammond Care Palliative and Supportive Care Service, Greenwich Hospital Sydney, Sydney, Australia

10.1136/bmjspcare-2012-000250.78

Severe Chronic Obstructive Pulmonary Disease (SCOPD) is associated with unpredictable deteriorations, morbidity and mortality, suggesting a role for advance care planning (ACP). Little is known about the practices and attitudes of respiratory physicians (RPs) to ACP. No tool to assess RPs practice of ACP was identified following a literature search. In developing a tool, items from several validated tools were combined. The resultant tool was assessed for face validity and piloted. $40 \mathrm{RPs}$ were invited to pilot the tool and $17(43 \%)$ responded. Over half (56\%) RPs discussed ACP with than less $50 \%$ of their current SCOPD patients. When discussions occurred, $80 \%$ reported they were "mostly" or "always" in the inpatient setting. While issues of diagnosis and incurability of COPD were commonly discussed, appointment of a health care proxy, the patients' values and goals and palliative care options were rarely addressed. ACP discussions led to understanding of patient preference for $69 \%$ of respondents who undertook these. Barriers to ACP included prognostic difficulties, patient and time related factors; facilitators included increasing patient frailty and willingness to discuss ACP. Many RPs (35\%) reported no formal training in ACP, but almost all reported learning ACP skills by observation and $80 \%$ were interested in further ACP education. This promising pilot suggests that ACP discussions SCOPD occur during acute inpatient care, rather than in advance. The responses and practices of RPs suggest 